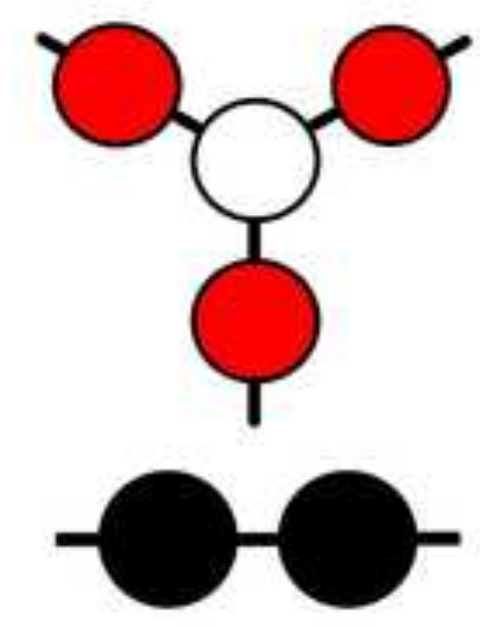

Reactants

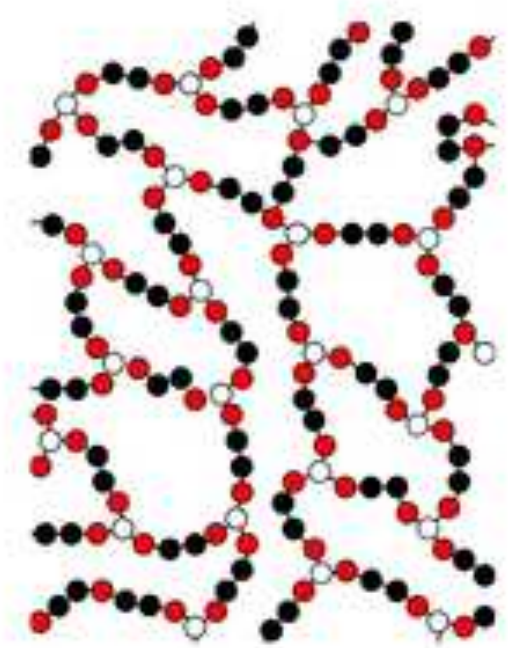

Densified Network

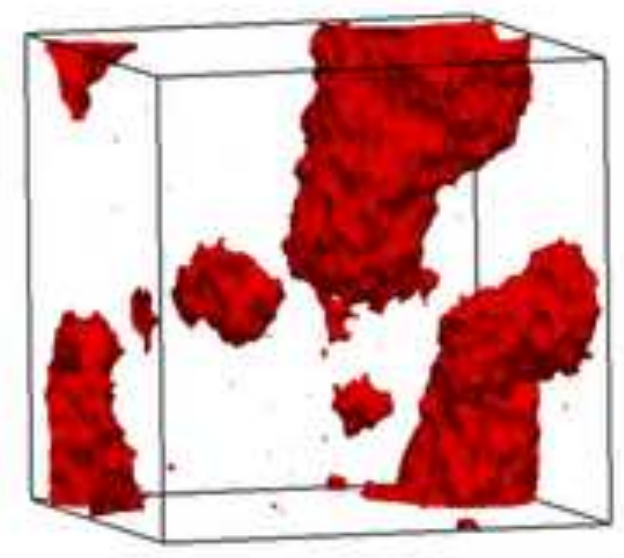

Cavitation 


\title{
Cavitation in crosslinked polymers: molecular dynamics simulations of network formation
}

\author{
Malia Zee ${ }^{\text {a }}$, Aaron J. Feickert ${ }^{\mathrm{b}}$, D. M. Kroll ${ }^{\mathrm{b}}$, and S. G. Croll ${ }^{*, a}$
}

a. Department of Coatings and Polymeric Materials, North Dakota State University, P.O. Box 6050, Dept 2760, Fargo, North Dakota 58108-6050, USA

b. Department of Physics, North Dakota State University, P.O. Box 6050, Dept 2755, North Dakota 58108-6050, USA

* Corresponding author. e-mail: Stuart.Croll@ndsu.edu; Tel: +1 7012319415.

Emails: Malia.Zee@ndsu.edu, Aaron.Feickert@ndsu.edu, Daniel.Kroll@ndsu.edu

\begin{abstract}
Crosslinked organic polymers are used in a wide variety of coatings and composites to distribute stress, increase toughness and protect the substrate by limiting the passage of aggressive chemicals. Enhancing performance of crosslinked polymers requires understanding how precursor chemistry and geometry, as well as crosslinking protocol, determine the structure and performance of the resulting network. Previous molecular dynamics studies have indicated that cavitation produces pores in simulated liquids, even metals (and the resultant solids), when there is only a single type of force, usually van der Waals, between particles. Here we show that nano-sized cavitation voids also occur in a system bound by van der Waals (Lennard-Jones) forces that is additionally crosslinked with strong covalent (FENE) bonds to form 3 or 6 functional solid networks. Cavitation was observed in both systems. These voids are not a consolidation of "free volume", nor due to a loss of volatiles, but happen as the solidification/cooling stresses exceed the local tensile strength of the material. At temperatures well above the glass transition temperature, "free volume" is distributed evenly throughout the sample in very small pores. As the system cools through its rubbery phase, a few larger voids form via cavitation. Although the loci of these larger voids is associated with crosslinked nodes, cavitation involves the rupturing of weak van der Waals (LennardJones) bonds between molecular chains in regions not constrained by the strong intramolecular bonds. Voids were observed to form during rapid quenches, as well as during much slower cooling at fixed
\end{abstract}


volume, which emulates adhesion of the network to a more rigid body. The voids are large compared to the dimensions of aggressive ionic species and water molecules, and may potentially reduce the barrier properties of a crosslinked coating or composite. Such pore formation, via cavitation, during network formation and curing is not incorporated in current theories of the crosslinking process.

Keywords: Crosslinked network, cavitation, pore, molecular dynamics

\section{Introduction}

Crosslinked polymers are used in many high-performance coatings such as polyurethanes, epoxies, and polyesters that decorate and protect a huge variety of infrastructure, engineering and consumer products, e.g. bridges, automobiles, pipelines, and aircraft. Polymers of similar composition are also used as adhesives and as the matrix in many reinforced composites, e.g. aircraft wings and fuselage, automobile parts, wind turbine blades, tennis racquets, boat hulls, etc. In all these applications, they provide adhesion, ensure binding of the ingredients, provide stress distribution and prevent corrosion or degradation of the reinforcing components or substrate. Typically, the crosslinked structure gives important advantages over thermoplastic polymers, including greater stiffness, wear resistance, improved chemical resistance, and better thermal stability. However, the period in service for which they provide good protection and performance is often disappointing, and a more thorough, detailed understanding of their structure is crucial for improving durability. Performance requirements and expectations demand that the network is fully formed, homogeneously crosslinked and continuous. Experimentally, it is difficult to determine how the distribution of crosslink junctions or network chains, or any other properties, vary through the bulk of a polymer, especially in coatings that are only 20 to $100 \mu \mathrm{m}$ thick and where the spacing between pigment or extender particles is much smaller yet. Simulating network 
formation using molecular dynamics approaches can provide necessary, additional information about property and structural variations at the nano- or molecular scale, and therefore provide insight into how crosslinked polymer performance might be improved.

In practice, crosslinked polymers are produced from two, or more, reactive liquid precursors. A huge variety of compositions are available to maximize performance of coatings in many different service conditions. Although the individual structure formed by a particular choice of reactive species is always of interest, it is also necessary to understand in more general ways how molecular architecture and topology affect the properties of three-dimensional polymer networks. Rather than focusing on a singular example of crosslinking reactants and their resultant network, we have used coarse-grained molecular dynamics to study general properties of network topology and structure.

The protective qualities of a polymer depend on its strength and whether it allows foreign species to permeate and attack a substrate. It is usually assumed that a higher degree of crosslinking leads to stronger, less permeable materials because the network structure is denser and will not allow foreign species to percolate. However, it is also recognized that aggressive species from the environment do, in fact, permeate through crosslinked polymers, and that the mechanisms involved are not well understood [1], making it difficult, a priori, to design a completely successful composition. A better understanding of the molecular and nanoscale causes of this permeability is needed in order to design materials with improved protective properties. However, traditional approaches to understanding crosslinked network formation focus on the overall crosslinked network, not on the possibility of void formation or other local variations [2-5]. Our interest in possible pore formation in crosslinked polymers that are formed from 
liquids is supported by simulations that show pores can arise by cavitation in amorphous liquids [6, 7], even metals [8].

Crosslinking reactions in organic polymers result in a final solid material that is denser than the initial liquid reactants. Shrinkage during polymer network formation is almost universal. Not only is a solid generally denser than a liquid, but many crosslinking reactions eliminate part of the original reactants as small, volatile reactants; it is also common for the initial liquid system to contain solvent that evaporates during the film formation. Thermal shrinkage is also common because many industrial applications involve curing at a temperature much higher than use temperature in order to assure the required chemical reactions are complete and occur within a reasonable time. Most coating polymers adhere to a substrate or pigments that are much more rigid. After crosslinking has proceeded to the extent that a molecular network spans its volume, it acquires solid-like properties (gels). At that point, the rigid substrate, or included particles, constrains densification and further reaction leads to internal strains within the network, in the plane of the substrate. In cases where the polymer forms without such rigid external constraints, it will be free to shrink, but even then it is common to find internal stresses and inhomogeneities in crosslinked polymer networks.

Although the molecular dynamics technique was developed over forty years ago, there have been comparatively few simulation studies of highly crosslinked polymer coatings. Simple, coarse-grained bead-spring models, which have been successfully used to treat bulk polymer melts and networks, have been used to study fracture and adhesion of highly crosslinked polymer networks [9 - 11]. Coarsegraining is useful because simulations are limited by computational resources; it allows simulations of networks on time scales on the order of microseconds and length scales on the order of tens of 
nanometers, which are necessary to study the mechanical response and network properties of these systems. Atomistic level simulations may include chemical and specific interactions but the same computer resources can only deal with much smaller systems and with times on the order of a few nanoseconds.

Crosslinked polymer networks are created from a wide range of molecules with different numbers and types of reactive sites. Early studies investigated crosslinked rubbers [12, 13], but typical coating or composite network polymers are more rigid and much more densely crosslinked. This study examines two systems to exemplify the range of possibilities within the higher crosslinked polymers. One is formed from a 3-functional "crosslinker" that reacts with a 2-functional chain-extender. The resulting network consists of 3-fold coordinated nodes, which is the minimum required to form a crosslinked network, connected by chains containing 4 beads. This is representative of networks formed from a wide variety of crosslinking chemistries that are used in coatings technology. The other exemplar employs a 6functional crosslinker that reacts with the same chain-extender and produces a much more densely crosslinked network. These two systems represent coatings and composites that are typically much more highly crosslinked than rubber networks, and thus this work complements the prior work of Grest and coworkers $[12,13]$ that described rubber-like networks.

Networks were formed at high temperatures and somewhat low densities to simulate the formation from liquid precursors. A volatile solvent is very common in coating formulations, but was not included here. The temperature of the resulting networks was subsequently reduced and, ultimately, they were investigated well into their glassy, solid state. Two scenarios were considered. In the first, the size of the simulation cell was held fixed as the network was cooled so that it suffered overall shrinkage strain, 
similar to what would occur in a network attached to a rigid substrate on curing. In the second, the system was cooled under zero load conditions, which corresponds to a bulk polymer with no rigid adherents. In practice, a polymer can flow and accommodate shrinkage while it is crosslinking, but still in the liquid, sol state, so most practical situations will fall in between the two procedures used here. In this work the emphasis is on understanding the general features of pore formation and obtaining an understanding of the network features that control, or permit, them.

\section{Models and Simulation Method}

The simple coarse-grained models used here are generated from precursors consisting of a 2-functional chain extender of two beads ('dimer' for brevity) and a 'crosslinker'. In coarse-grained models such as these, one bead represents not one atom but, rather, a group of atoms with the convenient result that network precursors have only a few beads per strand.

Following earlier work [9], the minimal case of two beads linked by a single spring is used for one of the reactants where each bead has one reactive site. The other reactants are two types of 'crosslinkers' that consist of several beads with different functionalities, in order to span a range of crosslinking densities and structures; see Figure 1. 


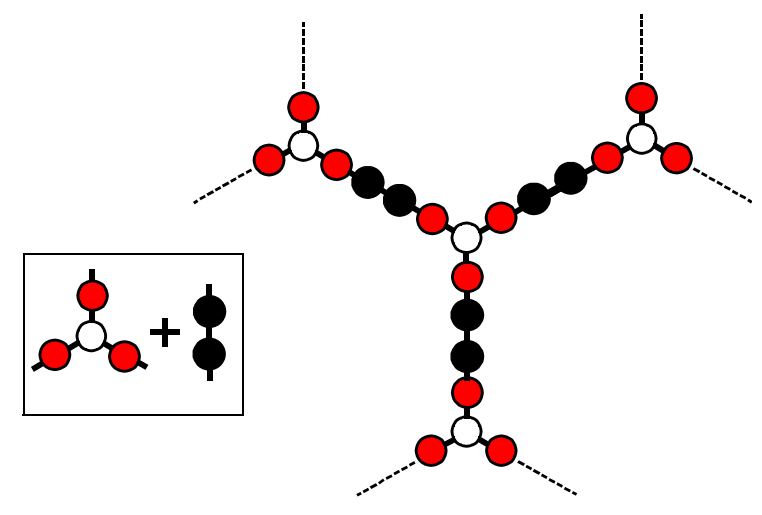

(a)
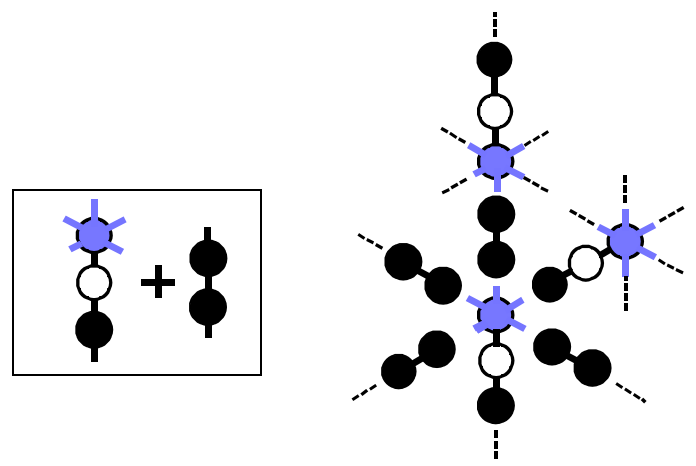

(b)

Figure 1. Two-dimensional representation of local network structure and precursor (a) 3-functional system, (b) 6-functional system. The precursors are shown in the inset boxes. Only one node of the 6-2 network is drawn completely due to the complexities of drawing a 6-functional network in two dimensions.

Functionalities are allowed to react according to the rule that black (as in Figure 1) must react with colored; no colored-colored or black-black reaction is allowed. The middle (open) beads do not react. Thus for the 3-functional system, a crosslinker molecule cannot react directly with another crosslinker molecule. For stoichiometry and $100 \%$ conversion, in the 3 -functional system each crosslinker (4 beads) shares 3 dimers ( 2 beads each) with other crosslinker molecules.

Table 1. Model Parameters

\begin{tabular}{|c|c|c|c|}
\hline Model & Crosslinker Molecules & 'Dimer' molecules & Total number of beads \\
\hline 3-functional & 1632 & 2448 & 11424 \\
\hline 6-functional & 1632 & 3264 & 11424 \\
\hline
\end{tabular}


In the other crosslinker, the 6-functional bead has one functionality already occupied by a two-bead tail, and that tail has a black bead that can react with one functionality on a separate crosslinker. Thus, this system needs only enough dimers for sharing between 4 functionalities of each crosslinker, i.e. 1632 x $4 / 2$ $=3264$ dimers.

Results presented here are for the simulations consisting of 11424 beads; these systems were generally large enough to produce results characteristic of the bulk, yet small enough to allow us study a large range of parameters in a reasonable amount of time. A small number of runs were performed using systems containing 1,428,000 beads (125 times larger) in order to check for finite size effects and verify the results obtained using the smaller networks.

Periodic boundary conditions and a rectangular parallelepiped simulation cell geometry were used to avoid boundary effects. All non-bonded beads interact via the cut-off and shifted Lennard- Jones (LJ) potential with a cutoff at $r_{c}=2.5 \sigma[12]$,

$$
\begin{aligned}
U(r) & =U_{L J}(r)-U_{L J}\left(r_{c}\right) & & \text { for } r \leq r_{c} \\
& =0 & & \text { for } r>r_{c}
\end{aligned}
$$

Where:

$U_{L J}(r)=4 \varepsilon\left[\left(\frac{\sigma}{r}\right)^{12}-\left(\frac{\sigma}{r}\right)^{6}\right]$

Here $\sigma$ is the length scale in the LJ potential, $r$ is the distance between bead centers and $\varepsilon$ is the energy parameter. This potential models the van der Waals attractive forces between all the beads and a strong 
repulsive core that defines the extent of the bead. "Covalent" bonds between beads, that were preexisting or formed during reaction, are described using a potential that prevents chain crossing [12]. This bond potential is the sum of the purely repulsive LJ interaction with a cutoff at $2^{1 / 6} \sigma$ (the minimum of the $\mathrm{LJ}$ potential) and a finite-extensible nonlinear elastic (FENE) attractive potential [13]:

$$
\begin{aligned}
U_{\text {FENE }}(r) & =-0.5 R_{0}^{2} k \log _{e}\left[1-\left(\frac{r}{R_{0}}\right)^{2}\right] & \text { for } r<R_{0} \\
& =\infty & \text { for } r \geq R_{0}
\end{aligned}
$$

where $k=30 \varepsilon / \sigma^{2}$ and $R_{0}=1.5 \sigma$. Here, $k$ controls the strength of the spring (bond) potential and $R_{0}$ is the maximum bond extension. This choice of parameters introduces incompatible length scales in the interand intra-polymer interactions which inhibit crystallization, allowing us to study amorphous materials. This choice also ensures relatively strong bonds and avoids chain crossing. The average bond length for these parameter choices is narrowly distributed around $0.961 \sigma$. The simulations are performed using the Large-scale Atomic/Molecular Massively Parallel Simulator (LAMMPS) [14].

All dimer and crosslinker beads have identical LJ parameters. Before crosslinking, the mixture of dimers and crosslinkers is equilibrated at a high temperature, $T=1.0 \varepsilon$, at zero load using a Nosé-Hoover thermostat and barostat with a time step of $0.005 \tau$, where $\tau=\sigma(m / \varepsilon)^{1 / 2}$ is the Lennard-Jones unit of time. After equilibration, the network is dynamically crosslinked under the same conditions. Every 2000 time steps $(10 \tau)$ potential bonding partners that have open functionalities and are separated by a distance less than $1.3 \sigma$ are identified, and the nearest potential bonding partners are crosslinked with a probability of $10 \%$. A detailed description of the crosslinking procedure can be found in other publications $[9,10,15]$ and the LAMMPS manual. This low probability allows the network to form fairly slowly and evenly. Crosslinking continued until a conversion $\sim 98 \%$ was achieved. Both systems were replicated five times to ensure that the behavior reported was typical. There proved to be only very small differences between replicas for each system, confirming that they were of sufficient size to produce reliable results, 
characteristic of a bulk system [9]. Results for the larger simulation size were consistent with the behavior of the 11,424 bead simulations.

Schematic diagrams of the reacted networks are shown in Figure 1. In one case, 3-functional crosslink nodes are connected by chains consisting of four beads [Figure 1(a)]. This system emulates many coating polymers with typical properties. The other system has a crosslinker of much higher functionality with only two beads between junctions and, in this form, has been used elsewhere [9, 10, 15]; it may also be thought of as being a very compact crosslinker with a functionality $f=6$ that has already reacted with one dimer. The notation for these two systems in discussion here will be ' $3-4$ ' and '6-2'. 4-functional systems, with both 2 and 4 beads between nodes, have also been investigated; they exhibit behavior intermediate between the systems reported here and do not provide additional insight into the phenomena discussed here.

\section{Results and Discussion}

The focus here is on the formation of voids within crosslinked polymer networks. However, understanding how and where the voids arise requires some background on the nature of the networks and their properties. These are dealt with first.

\subsection{Crosslinking}

Crosslinking reactions were continued until approximately $98 \%$ of all possible bonds were formed. This is higher than is generally achieved in practice or in the laboratory, but this value provides an easily 
repeatable reference point for further studies. The number density of the initial solution is $\rho=0.8 \sigma^{-3}$. Because of the comparatively short FENE bond length, the density of the systems increases while crosslinking, with $\rho 0.91 \sigma^{-3}$ and $0.95 \sigma^{-3}$ for the 3-functional and 6-functional systems, respectively, at $T$ $=1$ and $98 \%$ conversion.

\subsection{Network Defects}

A loose or dangling end occurs when a reactive site remains unused, e.g. a dimer bound to a crosslinker at only one end. Loose ends do not contribute to the elastic properties of a network. Their impact on permeability is probably more complicated: they are likely to provide less of a barrier to diffusion than dimers that are properly connected, while providing more of a barrier than a completely disconnected dimer (i.e., one that is not connected to the network at all). Another network defect considered is a minimal loop, which occurs when a dimer makes both bonds to a single crosslinker molecule. Although minimal loops may contribute to elastic and barrier properties via entanglements in less densely crosslinked systems, this is improbable here because of the short length of the dimers. To a first approximation, minimal loops are probably not network effective at all, in a manner analogous to dangling ends. In any case, there are few minimal loops in these models, so their contribution to the overall network effectiveness, if any, would still be negligible. To characterize the effective connectivity of each crosslink site, the numbers of bonds, minimal loops, and loose ends were determined for each crosslinker. The effective functionality was then determined using the relation:

[effective functionality $]=[$ bonds made $]-2[$ minimal loops $]-[$ loose ends $]$. 
The effective functionality is essentially the number of crosslinks to larger network fragments at each node, as a fraction of the nominal functionality. The effect of network defects in the 3-4 and 6-2 networks is shown in Figure 2. While the incidence of dangling ends was small for both models, the occurrence of loops increases with the functionality of the crosslinker, as might be expected. For this reason, the 3-4 model has a larger effective functionality than the 6-2 model.

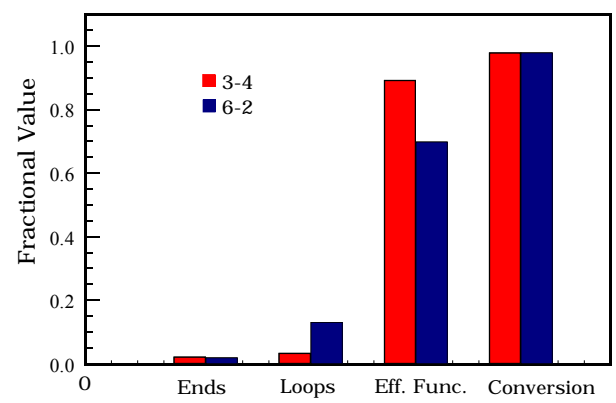

Figure 2. Fraction of dangling ends, loops, fractional effective functionality (eff. func.) and the fraction of possible bonds that formed (conversion) for the crosslinked networks.

Further examination showed that there were a few fragments of material that were not incorporated into the main network during crosslinking. Figure 3 contains representative diagrams of the network showing only the fragments. Crosslinking requires that reactive functionalities can approach each other closely enough to react. By chance, there will always be a small number that could not diffuse to encounter a suitable co-reactant, so that there will be a small number of clusters not connected to the network backbone, especially in stoichiometric systems. This mirrors practical experience since a small amount of material can often be leached out of crosslinked polymer networks using a suitable solvent $[16,17]$. For the systems described in Figure 3, $0.58 \%$ of the nodes in the 3-4 model and $0.6 \%$ of the nodes in the $6-2$ model were not linked to the network backbone. 


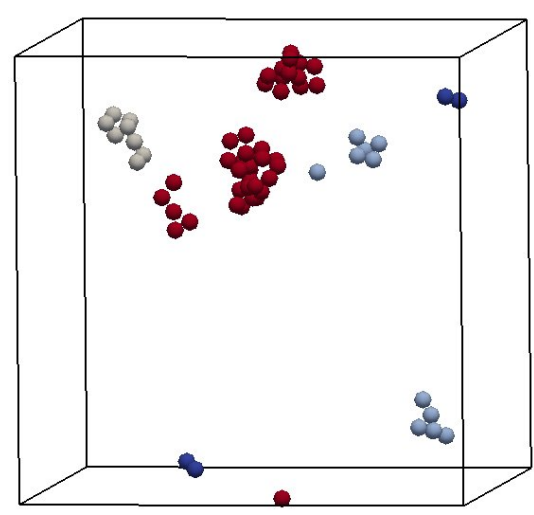

(a) 3-4 network

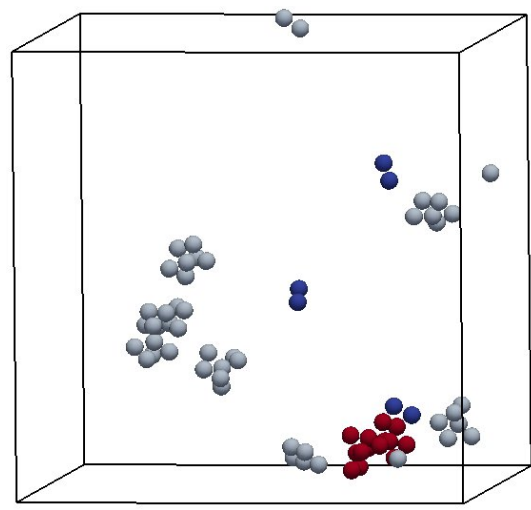

(b) 6-2 network

Figure 3. Position and size of fragments in the 3-4 and 6-2 systems after crosslinking. The conversion in the 3-4 network is 98.5\%; there are fragments containing between two and fourteen nodes. The conversion in the 6-2 network is $97.7 \%$ where there are slightly more fragments containing between two and fourteen nodes. Colors are used only to differentiate between separate clusters.

\subsection{Glass Transition Temperature}

To determine the glass transition temperature, $T_{g}$, simulations were performed at zero load using a NoséHoover thermostat and barostat. Temperature was lowered incrementally from $T=1.0$ at a rate of 0.1 reduced units per 400,000 time steps $(2,000 \tau)$. Data collected during the last 100,000 time steps of each stage were averaged in order to create the Volume-Temperature plots shown in Figure 4 from which the glass transition temperature was determined at the intersection of the straight lines fitted to the high and low temperature results.

The glass transition temperature is approximately 0.5 for both models; thus these simulations provide distinct, broad rubbery and glassy regimes. The results for the 6-2 system agree with the value obtained before [9]. 


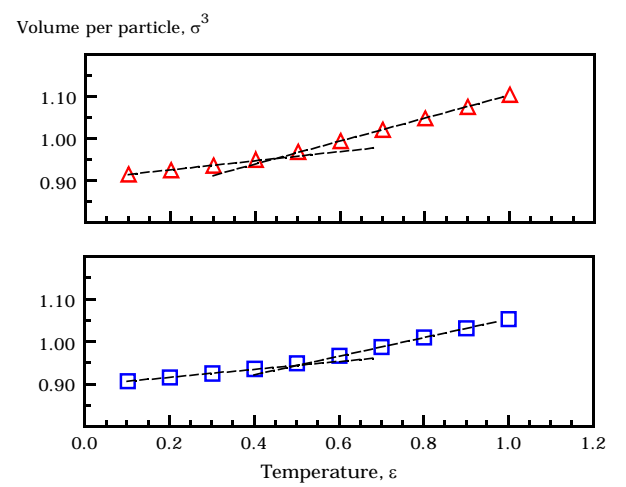

Figure 4. Specific volume as a function of the temperature. Top: 3-4 system, bottom: 6-2 system. The glass transition temperatures were identified as the intersection of the dashed lines. Values are given in Table 2.

\subsection{Elastic Moduli}

Calculations of elastic moduli above $T_{g}$ were made in order to confirm that the simulations of these two networks conformed to expectations for crosslinked polymers. Some results are listed in Table 2.

Table 2. $T_{g}$ and Young's moduli $(E)$ at $T=1.0$.

\begin{tabular}{|c|c|c|} 
System & $T_{g}\left(\varepsilon / k_{B}\right)$ & $E\left(\varepsilon / \sigma^{3}\right)$ \\
\hline $3-4$ & 0.448 & 0.19 \\
\hline $6-2$ & 0.502 & 1.12 \\
\hline
\end{tabular}

It can be seen that the higher functionality and shorter chains between crosslinks resulted in model 6-2 having a larger rubbery modulus than the 3-4 model, consistent with the predictions of affine rubber elasticity theory, which usually provides a reasonable estimate of the low-strain behavior of crosslinked polymers. 


\subsection{Void Formation}

It is not possible in a random dense network for all the beads to be positioned in their potential minimum. While many are, there will be a distribution of unoccupied, "free" volume where it is not the case. In the crosslinked networks at $T=1.0$, i.e. in the initial, hot, rubbery state, there are many small pores distributed randomly across the volume of the network; see Figure 5.

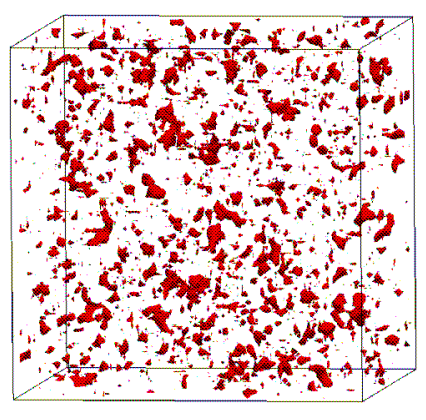

(a) 3-4 network

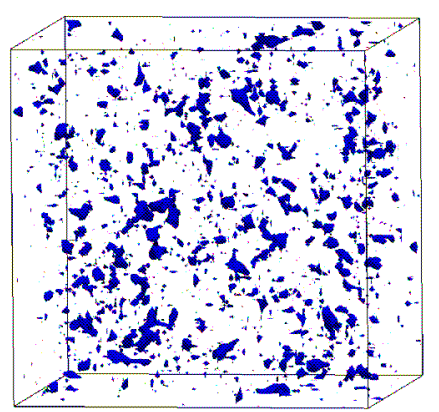

(b) 6-2 network

Figure 5. Pore space for (a) 3-4 and (b) 6-2 networks at $T=1.0$ after crosslinking at zero load. The isosurfaces are the loci of points a distance $0.8 \sigma$ from the nearest node of the network. The maximum node separations in these configurations are $1.08 \sigma$ for the 3-4 network and $1.11 \sigma$ for the $6-2$ network.

There appears to be little difference between free volume distributions in these two systems, with the more highly functional systems having slightly fewer but larger pores. Images in Figure 5 conform to what might be conventionally expected when the term "free volume" is used in discussions; there is no sign of cavitation under these conditions. Our interest here is to understand how the free volume distribution changes as the systems are cooled from the rubbery state into the glassy state. 
We have employed two distinct protocols during cooling in order to identify parameters which may influence both the distribution and magnitude of free volume. They represent limiting cases of what occurs in industrial thermosets which may be first oven-cured to obtain high conversions and then cooled to ambient temperatures for use. Coatings formed in this manner are confined in lateral dimensions by adhesion to their substrates, and shrinkage in the direction of thickness may also be restricted because it requires cooperative motion of polymer chains across long distances. Similarly, in composites, extensive shrinkage of the matrices is inhibited by the rigid, densely packed reinforcement fibers, and in pigmented coatings by rigid pigment particles. Such constraints are represented here by cooling at fixed volume (i.e., fixed cell dimensions). The second scenario is cooling at zero load (the simulation cell is allowed to shrink), which models a bulk polymer with no rigid constraints that is free to relax strains caused by cooling. We expect the behavior of real systems to be bracketed by the results for the two cases used here. We also study the evolution of the pore space when the networks, cooled at constant volume (constrained), are then allowed to relax under zero load. The effect of cooling rate in the various circumstances will be explained as the results are discussed.

Figure 6 shows the pore size distribution, defined as the probability distribution that a randomly chosen point in the simulation cell is a distance $d$ from the closest network node.

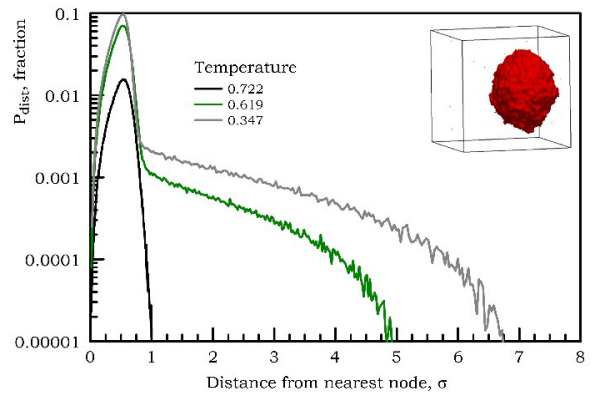

(a) 3-4 network

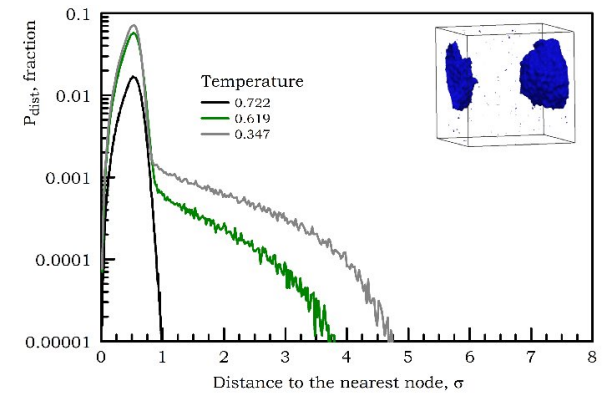

(b) 6-2 network 
Figure 6. Pore space distribution function $P_{d i s t}(d / \sigma)$. (a) 3-4 network: $P_{\text {dist }}(d / \sigma)$ at various temperatures for ramped cooling at fixed cell dimensions along an isochore. Insets in the diagrams are visualizations of the globular pores that form. The pore formation transition initiates at $T \approx 0.673$, well above the glass transition temperature, $T_{g}=0.448$. (b) 6-2 network: $P_{\text {dist }}(d / \sigma)$ at the same temperatures and color coding. The pore formation transition temperature is $T \approx 0.658$, well above the glass transition temperature, $T_{g}=$ 0.502 .

Initially, at high temperatures, $P_{d i s t}(d / \sigma)$ has a narrow peak near $0.5 \sigma$, i.e. approximately midway between beads that are close to the minimum in the FENE (bonded) potential $(0.961 \sigma)$ or the minimum for the LJ potential for non-bonded beads $(1.12 \sigma)$. These pore spaces at high temperature were shown in Figure 5. The distribution changes little as the temperature is lowered until, at $T \approx 0.65$, a large-separation tail in the distribution suddenly appears, indicating that a few open spaces have formed. The tail in the distribution for the 6-2 system, for example, indicates that a void has formed that has a radius greater than $4 \sigma$ at $T=$ 0.619. The tail in the distribution is somewhat larger in the 3-4 system, and in both cases, the tail increases in size upon further cooling, as expected. Visualizations of this pore space, shown as insets in figure 6 , indicate that the pores are globular in form. Pores are present, even though there is no possibility, in these simulations, of pores being left behind by the escape of volatile material.

Figure 7 shows the variation of the pressure (left panel) and van der Waals energy per particle (right panel) as a function of temperature for ramped cooling at fixed volume. As the temperature is lowered from $T=1.0$, the network shrinks and the pressure becomes increasingly negative (tensile) until the tensile strength of the van der Waals network is reached. At this point it ruptures and a void is formed, relieving some of the stress. 

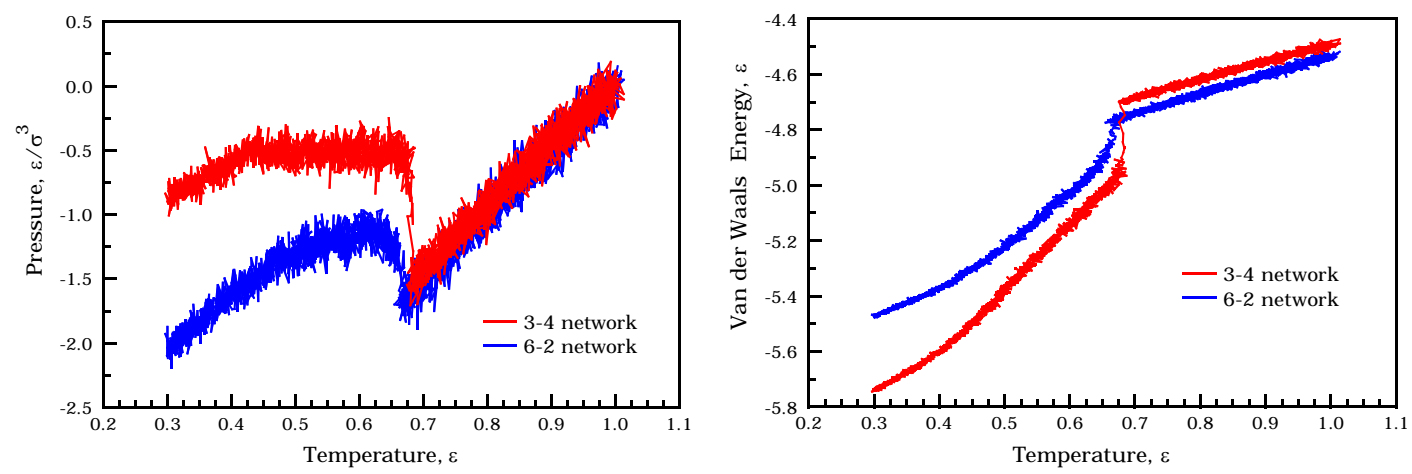

Figure 7. Pressure, $P$, (left panel) and van der Waals energy, $E_{v d W}$, per particle (right panel) as a function of temperature for ramped cooling at fixed volume. 3-4 (red) and 6-2 (blue) networks.

The fact that the surrounding crosslinked network does not rupture limits the possible stress relaxation. The pressure then stays relatively constant (and the void size increases) as the temperature decreases until the low temperature glassy phase is reached and the pressure starts to become more negative again. The behavior of the van der Waals energy per node as a function of temperature is consistent with this picture. In particular, it initially decreases linearly with temperature until dropping discontinuously at the voidformation transition. Below the transition, the density of the coherent regions of van der Waals network is greater than above the transition, resulting in significantly stronger binding energies. We find a transition temperature $0.673 \varepsilon$ for the $3-4$ network and $0.658 \varepsilon$ for the $6-2$ network. Because of the higher density of the 6-2 network, the critical pressure for the 6-2 network $\left(P^{*}=-1.71 \varepsilon / \sigma^{2}\right)$ is larger in magnitude than for the 3-4 network $\left(P^{*}=-1.53 \varepsilon / \sigma^{2}\right)$. Note that these transition temperatures are significantly higher than the glass transition temperatures for these networks. The results are summarized in Table 3.

Table 3. Cavitation temperature, $T^{*}$, and pressure, $P^{*}$, for fully crosslinked networks obtained when the temperature is ramped from $T=1.0$ to $T=0.3$ at constant volume over six million time steps. $\rho$ is the number density of each network. Results are averages taken over five independent replicas. 


\begin{tabular}{|c|c|c|c|c|c|} 
Network & $N$ & Conversion & Density, $\rho$ & $T^{*}, \varepsilon / k_{B}$ & $P^{*}, \varepsilon / \sigma^{2}$ \\
\hline $3-4$ & 11424 & 0.98 & 0.9075 & 0.673 & -1.53 \\
\hline $6-2$ & 11424 & 0.974 & 0.948 & 0.658 & -1.71 \\
\hline
\end{tabular}

The pore size and morphology depends on the rate of cooling. Figure 8 compares the pore size distributions at $T=0.3$ after a rapid quench (blue) and slow ramped cooling (red) starting from the same $T$ $=1.0$ crosslinked configuration. To generate the quenched configuration, velocity rescaling was used to quench the network to $T=0.3$. The simulation was then continued for 400,000 time steps using a Langevin heat bath algorithm. For the ramped cooling, the temperature was slowly lowered to $T=0.3$ over six million time steps using the Langevin heat bath algorithm. In both cases the simulations were performed at fixed volume. As might be expected, the resulting pore space is larger for ramped cooling, since the void had a longer time to anneal and grow above the glass transition temperature.

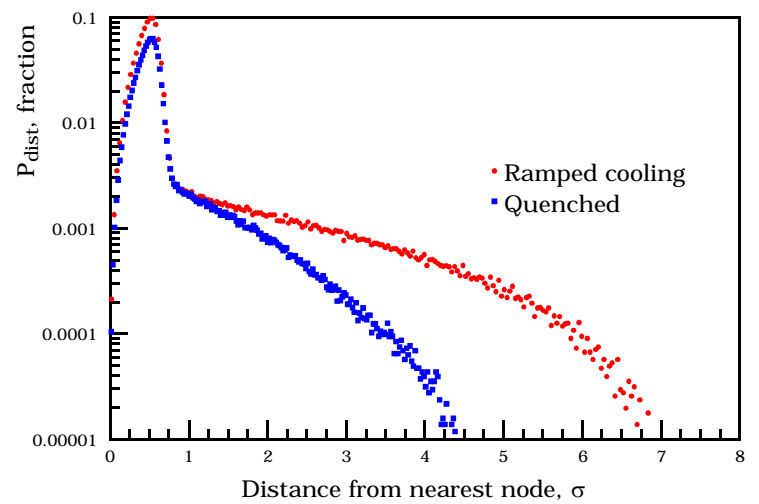

(a) 3-4 network

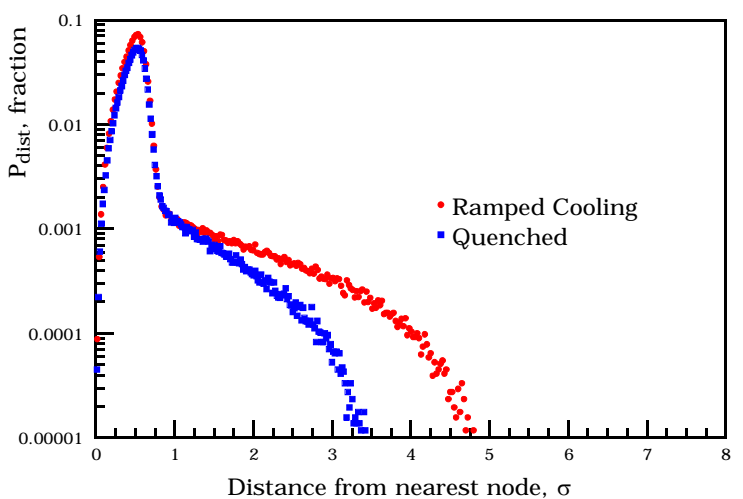

(b) 6-2 network

Figure 8. Pore space distributions $P_{\text {dist }}(d / \sigma)$ in both crosslinking systems at $T=0.3$ : contrasting a rapid quench (blue) with slow ramped cooling (red) from $T=1.0$, at fixed volume.

An interesting result is that the pore morphology is very different for the two cooling scenarios.

\subsection{Pore Morphology}


Figure 9 shows the pore space isosurfaces for the 3-4 and 6-2 networks at $T=0.3$ after a rapid quench. The globular pore shape for a slow ramped cooling from $T=1.0$, at fixed volume, is shown in the inset to figure 6 . The isosurfaces are the locus of points a distance $0.8 \sigma$ from the nearest node of the network. After a quench to below $T_{g}$, several pores have formed, and although their total volume is less than was found after a slow temperature ramp, the pores have a ramified structure that almost percolates across the simulation cell. It may not have been appreciated that such void structures were possible in a crosslinked network, but it is very important because the protective qualities of polymers used in coatings and composites, i.e. permeability as well as other properties, are obviously related to any such structural imperfections.
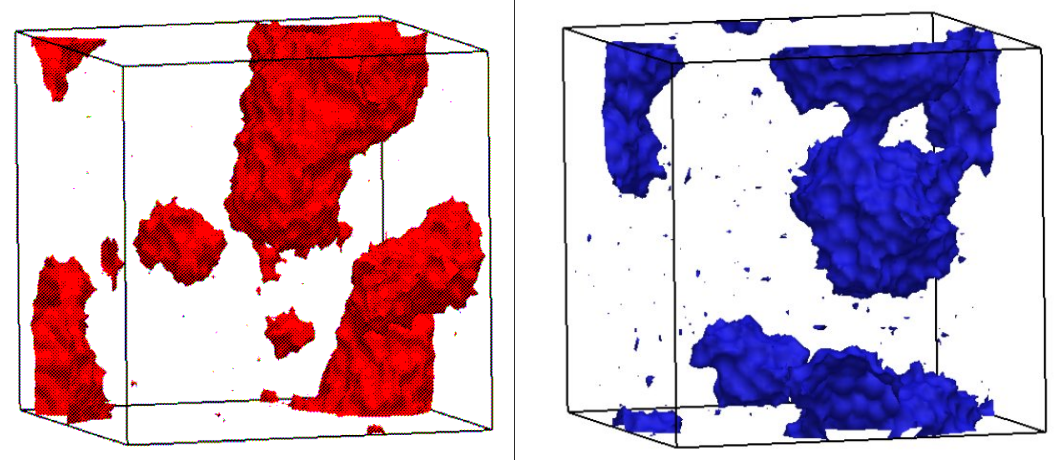

Figure 9. Pore space at $T=0.3$ after a rapid quench, left panel 3-4 network and 6-2 network, right panel. The isosurfaces are the locus of points a distance $0.8 \sigma$ from the nearest node of the network. Ramped cooling produces globular pores as seen in the insets to figure 6 .

A convenient measure of pore size is the reduced pore volume, $v_{p}$, quantified using the pore distribution functions plotted in Figure 8, with $d / \sigma$ greater than the given threshold values, and normalized by the simulation cell size. Results for $v_{p}$ at $T=0.3$ for pores formed using both cooling protocols are shown in Table 4. 
Table 4. Reduced pore volume, $v_{p}$, at $T=0.3$ after ramped cooling or a quench from $T=1.0$ for thresholds $d / \sigma=0.8$ and 1.0 .

\begin{tabular}{|c|cc|cc|}
\multicolumn{1}{|c|}{} & \multicolumn{2}{|c|}{ Ramped } & \multicolumn{2}{|c|}{ Quenched } \\
\hline Threshold, $d / \sigma$ & 0.8 & 1.0 & 0.8 & 1.0 \\
\hline$v_{p}, 3-4$ & 0.123 & 0.109 & 0.11 & 0.091 \\
\hline$v_{p}, 6-2$ & 0.084 & 0.072 & & 0.074 \\
\hline
\end{tabular}

What happens to the pores when the constant volume constraint is relaxed? Figure 10 compares the pore space distribution function, $P_{\text {dist }}(d / \sigma)$, after ramped cooling along an isochore (lines) and subsequent relaxation over one million time steps at zero load, with the fixed volume constraint removed (symbols). Data for $T=0.529,0.442$ and 0.347 are shown.

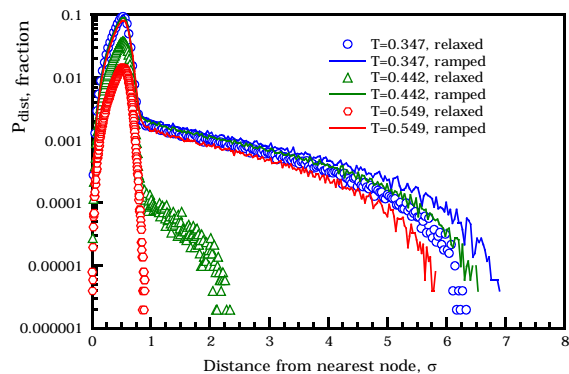

(a) 3-4 network

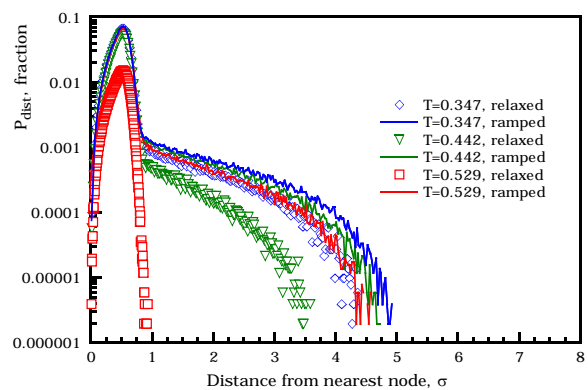

(b) 6-2 network

Figure 10. Comparison of the fractional pore space distribution function, $P_{d i s t}(d / \sigma)$, after ramped cooling along an isochore (solid lines) then subsequent relaxation over one million time steps at zero load (dashed lines). Data for $T=0.529,0.442$ and 0.347 are shown; $T_{g}=0.448$ for the $3-4$ network and 0.502 for the 62 network. Above the glass transition the network relaxes on this time scale and the pore space vanishes. Below $T_{g}$ relaxation becomes much slower as the temperature decreases and the pores shrink somewhat, but persist.

Above the glass transition the network relaxes on this time scale and the pore vanishes; below $T_{g}$ relaxation slows, as expected. Figure 11 collects these results and shows the reduced pore volume as a 
function of temperature. Again, one can see that the 3-4 system permits larger void volume than does the more densely functional system. Below the glass transition, decreased mobility results in greater residual pore volume. Correspondingly, the pores may persist below $T_{g}$ in real crosslinked polymer networks even in the absence of external mechanical constraints. Further, the voids are up to several bead diameters in size. Corresponding pores in an actual polymer network might be up to several nanometers in diameter and could contribute to the permeability of water and ions and serve as initiation sites for larger scale mechanical failure.
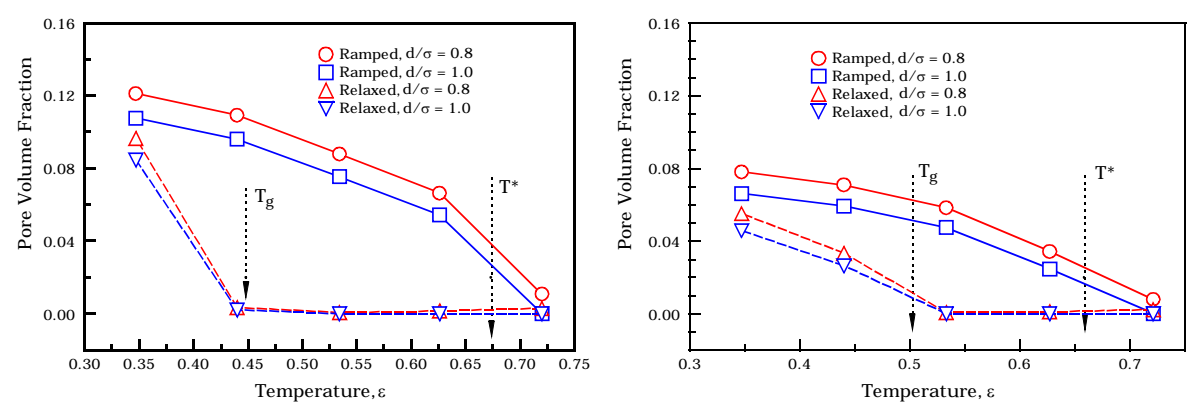

Figure 11. Reduced pore volume, $v_{p}$, i.e. pore volume beyond the threshold distance divided by the volume of the simulation cell, for thresholds $d / \sigma=0.8$ and 1.0 , as a function of temperature for the 3-4 system (left panel) and the 6-2 system (right panel) after ramped cooling at fixed volume and subsequent relaxation at zero load for one million time steps. Annealing at zero pressure for this length of time is sufficient to relax the network above the glass transition temperature.

\subsection{Cavitation in Crosslinked Networks}

Void formation by cavitation has been explored in a variety of simulations of liquids and materials in which there is only a single, general interaction between the components, e.g. polymer cavitation and crazing during deformation of thermoplastic polymers [6, 8, 18 - 23]. However, we find that pores appear even in a highly connected network of strong covalent (FENE) bonds in addition to LennardJones forces between all the beads. 
This appearance of the large pores is not a gradual consolidation of pore volume over a range of temperature, but a sudden occurrence at a particular temperature and pressure. For our current choice of models and parameters, pores form above the glass transition temperature; while this may not be a universal feature of crosslinking systems, it is important because many crosslinked polymers are used in a temperature range around or below $T_{g}$. Pore formation was seen in previous simulations of the 6-2 polymer system $[10,11]$, but unexplored there because the focus was on the mechanical properties of the crosslinked network. Void formation and cavitation in LJ liquids has been extensively studied [6, 7, 24 28] where it was found that void formation generally occurs at the spinodal and is held to be characteristic of liquids.

Qualitatively, the cavitation in these systems is easy to understand. As the temperature is lowered, the specific volume becomes smaller as the particles relax towards their potential minimum. At fixed volume, this causes the pressure to become negative, which can cause local cavitation when the tensile stress exceeds the mechanical strength of the material, resulting in a reduction of the total free energy. The density-pressure-temperature parameters at which cavitation voids form in van der Waals fluids [26, 29] are very similar to what we find in these crosslinked rubbery networks, which confirms that the voids in these crosslinked networks are indeed the result of cavitation. This, and the fact that the network backbone remains intact through the transition, indicates that the failure of the homogeneous LennardJones (van der Waals) bonding is responsible for void formation, even in crosslinked networks.

It may be difficult to imagine how such cavitation could occur in an homogeneously crosslinked network. However, the crosslinking process results in a network in which many nodes, that are spatial neighbors, are not bonded together. Thus there are potential fissures, of varied extent, across which only Lennard- 
Jones forces are present. In addition, the internal pressure is not homogenously distributed, but stress is concentrated at the crosslink nodes; cavitation appears to nucleate at such nodes next to a potential fissure. These few, irregular fissures are where voids can form in these networks, in close analogy to what happens in pure Lennard-Jones systems.

These results indicate van der Waals network failure and void formation should occur in a wide range of materials as they are cooled under conditions where local stresses cannot relax. We find voids form during network formation and solidification, prior to any in-service stresses. If that is true, they are likely initiation sites for larger failures during service, especially if the system cannot relax to eliminate the voids because the prevailing temperature is below the glass transition temperature. This would also be true of their likely impact on permeability in protecting the reinforcing elements of a composite or the substrate of a coating. It is notable that significant voids appear even in the highly crosslinked system modeled here. Solely increasing network density may not diminish permeability enough to improve barrier properties, but controlling the glass transition temperature and compatibility of the network with the possible permeants must also be considered.

\section{Conclusions}

In studying crosslinked networks using a typical coarse grained molecular dynamics approach, void formation and growth is found that initiates above the glass transition temperature of the network as it cools through its rubbery to its glassy low temperature state. It is somewhat surprising that these cavitation voids occur in simulations of crosslinked polymers where the network is supported by strong directional, FENE (covalent) bonds as well as the much weaker, non-directional Lennard-Jones (van der Waals) interactions. Such voids have been noted and studied in simulations of liquids and amorphous 
solids with only Lennard-Jones forces, at very similar values of pressure, temperature and density. These voids are not a consolidation of "free volume", nor due to a loss of volatiles, but are caused by cavitation, happening as the solidification/cooling stresses exceed the local tensile strength of the material. The voids first appear in the rubbery state while the network has substantial mobility. That voids occur during the formation of crosslinked networks seems to be a new observation that has consequences for the enduse properties of such materials and their long term performance. Although these finding are very consistent with previous simulations of liquids, the time scales of all such simulations is very brief and it may be that the pores will disappear in real liquids and solids if they are well above their glass transition temperature and are not constrained by adhesion to, or between, more rigid material bodies. This is currently under study, where these effects in much larger and more extended simulations are investigated. However, pores are much more likely to persist if the polymer network is maintained near or below the glass transition, and constrained by adhesion. This is the case for most polymer coatings.

The simulations show that the network connectivity, network strength and internal pressure are all heterogeneous. Polymers formed from mobile reactive precursors produce clusters of reacted material as the network grows towards the gel point. It follows that there can be adjacent regions that are not connected by covalent bonds and are weak points where voids can form to reduce the internal tensile strain caused by the solidification/cooling process. These voids, depending on circumstances, may comprise a significant fraction of the simulation volume, and their dimensions would scale to the order of nanometers in a physical system. This suggests that there may be regions of lowered density, as seen in recent results [30]. These would occur especially in polymers that are confined by adhesion to substrates, reinforcing fibers or pigment particles, and are in use around or below their glass transition temperature. Not only may the voids be the initiation sites of larger scale mechanical failure, but they are likely to contribute to the permeability of the polymer, especially if degradation in service acts to enlarge such features. 


\section{Acknowledgements}

Funding for this research came from the Lowell Woods Foundation at North Dakota State University and the Army Research Laboratory. Computer access, financial, and administrative support from the North Dakota State University Center for Computationally Assisted Science and Technology and the U.S. Department of Energy through Grant No. DESC0001717 are gladly acknowledged.

\section{References}

1. Taylor, S.R.; Contu, F.; Santhanam, R.; Suwanna, P. The use of cationic fluorophores to characterize ionic pathways in organic coatings. Prog. Org. Coatings 2012, 73, 169-172.

2. Gordon M., Good's Theory of Cascade Processes Applied to the Statistics of Polymer Distributions, Proc. Roy. Soc. 1962, A268, 240 - 256

3. Dušek K; Duškova'-Smrčková $\mathrm{M}$, Network structure formation during crosslinking of organic coating systems, Prog. Polym. Sci. 2000, 25, 1215-1260

4. Miller D. R.; Macosko C. W, “A New Derivation of Post Gel Properties of Network Polymers, Macromolecules, 1976, 9(2), 206 - 211

5. Kiil S, Quantification of simultaneous solvent evaporation and chemical curing in thermoset coatings, J. Coat. Technol. Res., 2010, 7 (5) 569-586

6. Sastry, S; Debenedetti P.G; Stillinger F. H., Statistical geometry of particle packings. II. "Weak spots" in liquids, Phys. Rev. E, 1997, 56(5), 5533- 5543 
7. Baidakov V. G.; Bobrov K. S., Spontaneous cavitation in a Lennard-Jones liquid at negative pressures, J. Chem. Phys., 2014, 140, 184506

8. Cai Y; Wu H. A.; Luo S. N, Cavitation in a metallic liquid: Homogeneous nucleation and growth of nanovoids, J. Chem. Phys., 2014, 140, 214317

9. Stevens, M.J. Interfacial fracture between highly cross-linked polymer networks and a solid surface: effect of interfacial bond density. Macromolecules 2001, 34, 2710-2718.

10. Tsige, M.; Stevens, M.J., Effect of Cross-Linker Functionality on the Adhesion of Highly CrossLinked Polymer Networks: A Molecular Dynamics Study of Epoxies. Macromolecules 2004, 37, 630637.

11. Tsige, M; Lorenz, C.D.; Stevens, M.J. Role of network connectivity on the meichanical properties of highly cross-linked polymers. Macromolecules 2004, 37, 8466.

12. Kremer, K.; Grest, G. In Monte Carlo and Molecular Dynamics Simulations in Polymer Science; Binder, K., Ed.; Oxford: New York, 1995; Chapter 4, pp 194-271.

13. Grest, G.S.; Kremer, K. Statistical Properties of random crosslinked rubbers. Macromolecules 1990, 23, 4994-5000.

14. Plimpton, S. Fast Parallel Algorithms for Short-Range Molecular Dynamics. J. Comp. Phys. 1995, 117, 1-19.

15. Stevens, M.J. Manipulating Connectivity to Control Fracture in Network Polymer Adhesives. Macromolecules 2001, 34, 1411-1415. 
16. Duarte, R.G.; Gonz'alez, S.; Castela, A.S.; Ferreira, M.G.S.; Souto, R.M. Sensing polymer inhomogeneity in coated metals during the early stages of coating degradation. Prog. Org. Coatings 2012, $74,365-370$.

17. Tsang, J.-S., Erhardt D. Current Research on the Effects of Solvents and Gelled and Aqueous Cleaning Systems on Oil Paint Films. J. Am. Inst. Cons. 1992, 31, 87-94.

18. Estevez, R.; Long, D. Probing and characterizing the early stages of cavitation in glassy polymers in molecular dynamics simulations Modelling Simul. Mater. Sci. Eng. 2011, 19045004.

19. Sixou, B. Molecular dynamics simulation of the first stages of the cavitation process in amorphous polymers Mol. Simul. 2007, 33, 965-973.

20. Rottler, J.; Robbins, M. Yield conditions for deformation of amorphous polymer glasses Phys. Rev. E 2001, 64, 051801.

21. Baljon, A.R.C.; Robbins, M. Simulations of Crazing in Polymer Glasses: Effect of Chain Length and Surface Tension Macromolecules 2001, 34, 4200-4209.

22. Mahajan, D.K.; Singh, B.; Basu, S. Void nucleation and disentanglement in glassy amorphous polymers Phys. Rev. E 2010, 82, 011803.

23. Baidakov V. G.; Bobrov K. S, Spontaneous cavitation in a Lennard-Jones liquid at negative pressures, J. Chem. Phys. 2014, 140, 184506.

24. Voloshin, V.P.; Naberukhin, Yu.I. Homogeneity loss phase transition in packings of LennardJones atoms under density decreasing. J. Mol. Liquids 1999, 82, 19-26.

25. Corti, D.S.; Debenedetti, P.G.; Sastry, S.; Stillinger, F.H. Constraints, metastability, and inherent structures in liquids Phys. Rev. E 1997, 55, 5522-5534. 
26. Kuksin, A.Yu.; Norman, G.E.; Stegailov V.V. The phase diagram and spinodal decomposition of metastable states of Lennard-Jones system High Temp 2007, 45, 37-48.

27. Sastry, S. Liquid limits: Glass transition and liquid-gas spinodal boundaries of metastable liquids Phys. Rev. Lett. 2000, 85, 590-593.

28. Baidakov V.; Protsenko, S. Singular Point of a System of Lennard-Jones Particles at Negative Pressures Phys. Rev. Lett. 2005, 95, 015701.

29. Debenedetti. P.G.; Truskett, T.M. The statistical geometry of voids in liquids. Fluid Phase Equilibria 1999, 158-160, 549-556,

30. Hughes A; Trinchi A; Chen F. F; Yang Y. S; Cole I. S; Sellaiyan S; Carr J; Lee P. D; Thompson G. E; T. Xia Q, The application of multiscale quasi 4D CT to the study of SrCrO4 distributions and the development of porous networks in epoxy-based primer coatings, Progress in Organic Coatings, to be published. 